\title{
Frequency-domain differential photoacoustic radar: theory and validation for ultrasensitive atherosclerotic plaque imaging
}

Sung Soo Sean Choi

Bahman Lashkari

Andreas Mandelis

Jungik Son

Natasha Alves-Kotzev

Stuart F. Foster

Mark Harduar

Brian Courtney 


\title{
Frequency-domain differential photoacoustic radar: theory and validation for ultrasensitive atherosclerotic plaque imaging
}

\author{
Sung Soo Sean Choi, ${ }^{a}$ Bahman Lashkari, ${ }^{a}$ Andreas Mandelis, ${ }^{a, k}$ Jungik Son, ${ }^{b}$ Natasha Alves-Kotzev, ${ }^{b}$ \\ Stuart F. Foster, ${ }^{b}$ Mark Harduar, ${ }^{c}$ and Brian Courtney ${ }^{b, c}$ \\ aUniversity of Toronto, Center for Advanced Diffusion-Wave and Photoacoustic Technologies, Department of Mechanical and Industrial \\ Engineering, Toronto, Ontario, Canada \\ bSunnybrook Research Institute, Physical Sciences Department, Toronto, Ontario, Canada \\ ${ }^{\circ}$ Conavi Medical, Toronto, Ontario, Canada
}

\begin{abstract}
Lipid composition of atherosclerotic plaques is considered to be highly related to plaque vulnerability. Therefore, a specific diagnostic or imaging modality that can sensitively evaluate plaques' necrotic core is desirable in atherosclerosis imaging. In this regard, intravascular photoacoustic (IVPA) imaging is an emerging plaque detection technique that provides lipid-specific chemical information from an arterial wall with great optical contrast and long acoustic penetration depth. While, in the near-infrared window, a 1210-nm optical source is usually chosen for IVPA applications since lipids exhibit a strong absorption peak at that wavelength, the sensitivity problem arises in the conventional single-ended systems as other arterial tissues also show some degree of absorption near that spectral region, thereby generating undesirably interfering photoacoustic (PA) signals. A theory of the high-frequency frequency-domain differential photoacoustic radar (DPAR) modality is introduced as a unique detection technique for accurate and molecularly specific evaluation of vulnerable plaques. By assuming two low-power continuous-wave optical sources at $\sim 1210$ and $\sim 970 \mathrm{~nm}$ in a differential manner, DPAR theory and the corresponding simulation/experiment studies suggest an imaging modality that is only sensitive and specific to the spectroscopically defined imaging target, cholesterol. $\odot$ The Authors. Published by SPIE under a Creative Commons Attribution 4.0 Unported License. Distribution or reproduction of this work in whole or in part requires full attribution of the original publication, including its DOI. [DOI: 10.1117/1.JBO.24.6.066003]
\end{abstract}

Keywords: differential photoacoustic imaging; endoscopy; image enhancement; atherosclerosis.

Paper 190080R received Mar. 20, 2019; accepted for publication May 21, 2019; published online Jun. 13, 2019.

\section{Introduction}

Atherosclerosis is a chronic cardiovascular disease (CVD) that is characterized by inflammation and the gradual buildup of lipid-rich plaque in the inner lining of the arterial walls (intima). ${ }^{1-4}$ While the full risk factors or the cellular mechanism of plaque development are not yet fully understood, early atherosclerosis is initiated as lipid-carrying low-density lipoproteins (LDLs) get retained beneath the endothelial cells of the vessels. ${ }^{2-4}$ The retained LDLs get oxidized, and endothelial cells trigger the immune response by attracting monocyte-derived macrophages to the sites. ${ }^{2-4}$ When the ingested lipids are not properly removed from macrophages by high-density-lipoproteins (HDLs), the internal level of the oxidized LDLs in macrophages increases abnormally and they transform into foam cells. ${ }^{2-4}$ These foam cells eventually rupture causing local damage to the endothelial cells. More inflammatory processes then follow to repair the injured endothelium by local cellular remodeling. ${ }^{3,4}$ Cellular remodeling, however, may end up with some degree of misrepair; therefore, the corresponding local regions only become more prone to plaque formation. As more of these events occur, the development of plaque self-accelerates, and its size increases over time. ${ }^{2-4}$

*Address all correspondence to Andreas Mandelis, E-mail: mandelis@mie .utoronto.ca
Although it may show no sign of disease for decades, atherosclerosis becomes dangerous when plaque rupture releases thrombogenic contents into the blood stream. This may result in partial or complete occlusion of the lumen, instigating cardiac events such as myocardial infarction, stroke, and death. ${ }^{1-8}$ Plaques with higher risk of rupture are referred to as vulnerable, and, for a subtype known as thin-capped fibroatheromas, their vulnerability is known to be highly correlated with the lipid composition of the plaques. ${ }^{6-8}$ To this end, techniques for in-vivo characterization of plaque lipid composition are sought. However, currently available modalities for vulnerable atherosclerosis plaque characterization exhibit several limitations. Angiography may detect plaque indirectly by locating stenosis and luminal irregularities but substantially lacks morphological or compositional information of plaques other than the extent of calcification. ${ }^{3,6}$ Intravascular ultrasound (IVUS) provides reliable information regarding plaque burden, but it fails to deliver information that reliably differentiates between various forms of soft plaque. ${ }^{3,9-11}$ Intracoronary near-infrared spectroscopy can sense plaque by its chemical composition, but it does not provide an accurate assessment of the depth profile of a plaque within the vessel wall. ${ }^{3,9,10}$ While intravascular optical coherence tomography (OCT) can present the plaque morphology with great resolution and characterize their lipid composition with high sensitivity based on the highly attenuating optical properties of lipids, its penetration depth is highly limited to about $1 \mathrm{~mm}$ due to severe light scattering through heterogeneous media. Furthermore, OCT requires an extra step of flushing the 
vessel with an optically clear medium, such as a contrast agent, before performing image acquisition as the OCT light does not penetrate well through blood. .,12,13 $^{2}$

Laser photoacoustics (PA) is a hybrid optical-acoustic imaging technology in which a subsurface target is excited with near-infrared light and imaged with optically induced ultrasound (US) signals. Satisfying both high optical contrast and high ultrasonic depth penetration, intravascular photoacoustics (IVPA) is emerging as a competitive modality for plaque detection. ${ }^{5-8}$ While high-power pulsed lasers have been the main optical sources for conventional PA imaging, ${ }^{5-8}$ a PA imaging modality has also been under intense development based on frequency-modulated (chirped) optical excitation with lowpower continuous-wave (CW) lasers and frequency-domain (FD) signal processing to obtain depth-resolved images of tissue chromophores. ${ }^{14-16}$ The technology is called the photoacoustic radar (PAR) and provides several unique imaging features including (1) efficient noise filtering and high signal-to-noise ratio (SNR) with low power irradiation; (2) micrometer axial resolution; (3) depth information, mapped into the PA spectrum; (4) physiological information based on the target's optical properties; and (5) reliable measurements based on two complementary channels of amplitude and phase. ${ }^{14-17}$ Furthermore, the use of CW optical sources in PAR allows flexible waveform engineering on the modulating optical signals that can lead to several unique or enhanced imaging features. From this perspective, our earlier introduction of single-frequency wavelength-modulated differential spectroscopy ${ }^{18-20}$ leads to the present multifrequency intravascular differential PA radar (IV-DPAR) system as an excellent example of the many waveform engineering possibilities for further advances in atherosclerosis imaging, beyond the physical limitations of the conventional PAR and pulsebased PA counterparts. The block diagram of the FD PAR data-processing sequence is described in Fig. 1.

\section{Theory of FD IV-DPAR}

The IV-DPAR may be considered an extension of the singleended PAR system where many imaging parameters are carefully engineered for the specific purpose of atherosclerotic plaque detection. Consequently, IV-DPAR inherits general traits and characteristics of PAR in terms of signal generation and processing. The novelty of IV-DPAR is the use of a second wavelength, $\lambda_{2}$, in real time with identical chirp modulation at a specific optical phase difference, $\varphi_{\text {optical }}$. The relationship between the two modulating optical signals, $r_{\lambda 1}(t)$ and $r_{\lambda 2}(t)$, are then expressed as ${ }^{21}$

$r_{\lambda_{1}}(t)=\sin \left(2 \pi f_{1} t+\frac{\pi B W_{c h}}{T_{c h}} t^{2}\right), \quad-\frac{T_{c h}}{2} \leq t \leq \frac{T_{c h}}{2}$,

$$
\begin{aligned}
r_{\lambda_{2}}(t)= & \sin \left(2 \pi f_{1} t+\frac{\pi B W_{c h}}{T_{c h}} t^{2}+\varphi_{\text {optical }}\right), \\
& -\frac{T_{c h}}{2} \leq t \leq \frac{T_{c h}}{2}
\end{aligned}
$$

where $f_{1}$ is the starting frequency, $B W_{c h}$ is the bandwidth, and $T_{c h}$ is the total length of the modulating chirps. When the two coherent optical waves, $r_{\lambda 1}(t)$ and $r_{\lambda 2}(t)$, are simultaneously absorbed by an identical absorber, the two PA waves are generated. Since the optical absorption coefficient of an absorber, $\mu_{a}$, and corresponding PA signal generation process are wavelength-dependent, the two resulting raw PA signals, $\mathrm{s}_{\lambda 1}(t)$ and $s_{\lambda 2}(t)$, may exhibit different amplitude and phase, but still they are coherent with a certain acoustic phase difference, $\varphi_{\text {acoustic }}$, as $^{21}$

$$
\begin{aligned}
s_{\lambda_{1}}(t)= & A_{\lambda_{1}}\left[\sin \left(2 \pi f_{1} t+\frac{\pi B W_{c h}}{T_{c h}} t^{2}\right)\right]+\mathrm{Z}, \\
& -\frac{T_{c h}}{2} \leq t \leq \frac{T_{c h}}{2} \\
s_{\lambda_{2}}(t)= & A_{\lambda_{2}}\left[\sin \left(2 \pi f_{1} t+\frac{\pi B W_{c h}}{T_{c h}} t^{2}+\varphi_{\text {acoustic }}\right)\right]+\mathrm{Z}, \\
& -\frac{T_{c h}}{2} \leq t \leq \frac{T_{c h}}{2},
\end{aligned}
$$

where $\varphi_{\text {acoustic }}=\varphi_{\text {optical }}+\varphi_{\text {shift }}$.

Here, $\varphi_{\text {shift }}$ is the relative phase shift between two waves (including both intrinsic and random phase shifts) during light-to-sound PA energy conversion. Here, $A$ is the amplitude of $s(t)$ that is affected by $\mu_{a}$ and the optical fluence at each wavelength, and $Z$ is the potential additive system noise. These two acoustic waves are related to each other by spatial and temporal constants and undergo stationary interference, resulting in a single differential PA signal upon generation. Depending on the selection of optical wavelengths and the initial $\varphi_{\text {optical }}$ between them, information carried by the resulting differential PA signals may vary dramatically, but a general form of raw differential PA signals can be described as ${ }^{21}$

$s_{\text {Diff }}(t)=s_{\lambda_{1}}(t)+s_{\lambda_{2}}(t)$.

In other words, the contents of the differential PA signal are determined by intrinsic interferences between two corresponding single-ended PA signals in the acoustic domain. Therefore, the parameters in IV-DPAR need to be carefully selected and calibrated to encode desired information in this differential PA channel. ${ }^{21}$

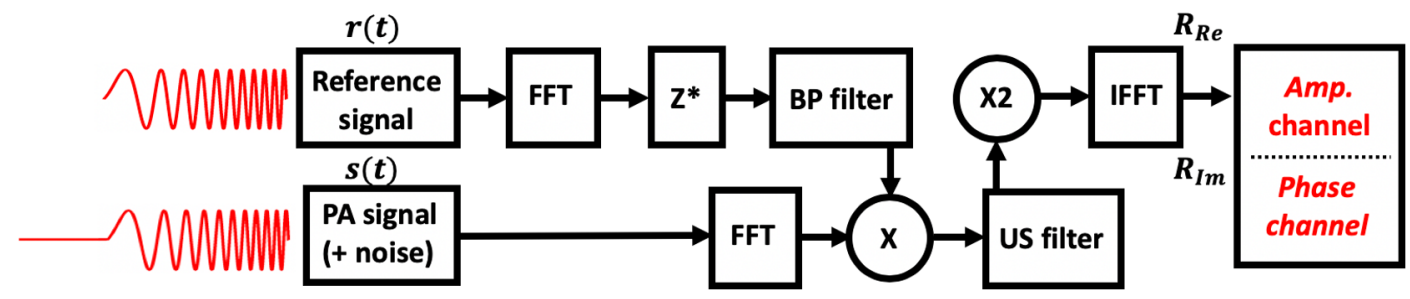

Fig. 1 Block diagram of the matched filter cross correlation and the pulse compression algorithms of FD IV-DPAR. FFT, fast Fourier transformation; IFFT, inverse fast Fourier transformation; $Z^{*}$, complex conjugate; Re, real component; Im, imaginary component; BP filter, bandpass filter; US filter, unit-step filter; and $\mathrm{X}$, multiplication. 
The main purpose of IV-DPAR in this particular application terol or the necrotic core of atherosclerotic plaques in arteries. Therefore, the resulting PA signals, at the time of detection, need to contain only the cholesterol information without any contribution from other types of arterial tissue. Based on the nearinfrared absorption spectra of various biological tissues found in human atherosclerotic arteries, ${ }^{6}$ this condition cannot be met at a single wavelength (i.e., $\lambda_{1}=\sim 1210 \mathrm{~nm}$ ) because the absorption peak of lipids overlaps with those of other atherosclerotic tissues. This may explain why conventional singleended pulse-based IVPA systems or even PAR cannot be reliable diagnostic methods for this application. Instead, intrinsic suppression of those undesirable contributions from other tissues may be achieved by employing the second wavelength at $\lambda_{2}=\sim 970 \mathrm{~nm}$. Within the wavelength range considered, $\lambda_{1}$ corresponds to the second vibrational overtone of $\mathrm{C}-\mathrm{H}$ bonds in cholesterol molecules. This absorption peak is, therefore, distinctively large near $\lambda_{1}$, while the lipid absorption below $\lambda=$ $\sim 1150 \mathrm{~nm}$ may be considered negligible. Two main peaks of normal arterial tissues are at $\sim 970$ and $\sim 1180 \mathrm{~nm}$, which correspond to the vibrational overtones of oxygen-hydrogen $(\mathrm{O}-\mathrm{H})$ bonds in water molecules. ${ }^{6}$ By utilizing their spectral relationships, IV-DPAR may cancel the contributions from undesirable noise by means of complete destructive interference is to improve detection sensitivity and specificity toward choles-

while being highly selective to cholesterol molecules only. Regarding the wavelength selection, it should be noted that the differential PA modality in general is flexible in terms of defining its detection targets. The IV-DPAR, in this application, focuses on evaluating cholesterol contents of plaques, and, therefore, $\lambda_{1}=\sim 1210 \mathrm{~nm}$ and $\lambda_{2}=\sim 970 \mathrm{~nm}$ are shown to be optimal. However, by selecting other sets of appropriate wavelengths, other important biological tissues can be readily focused on.

\section{Materials and Methods}

\subsection{Simulation Parameters}

Among various possible types of optical modulation waveforms, a square waveform was shown to exhibit the highest SNR in PAR due to the difference in the energy content and frequency spectrum of the excitation signal involved in PA signal generation. ${ }^{22}$ For this reason, the following linear-frequency modulation (LFM) square chirps were chosen to modulate the two optical sources for IV-DPAR ${ }^{21}$

$$
I_{1}(t)=A_{1}\left\{0.5 \operatorname{sgn}\left[\sin \left(2 \pi f_{1} t+\frac{\pi B_{c h}}{T_{c h}} t^{2}\right)\right]+0.5\right\}
$$

$I_{2}(t)=A_{2}\left[0.5 \operatorname{sgn}\left(\begin{array}{c}{\left[\sin \left(2 \pi f_{1} t+\frac{\pi B_{c h}}{T_{c h}} t^{2}\right) \cos \left(\frac{\varphi_{\text {optical }}}{180} \pi\right)\right]} \\ +\left\{\mathcal{H}\left[\sin \left(2 \pi f_{1} t+\frac{\pi B_{c h}}{T_{c h}} t^{2}\right)\right] \sin \left(\frac{\varphi_{\text {optical }}}{180} \pi\right)\right\}\end{array}\right]+0.5\right]$ where $I_{1}$ and $I_{2}$ are the optical intensities and $A_{1}$ and $A_{2}$ are the arbitrary amplitudes of the $1210-\mathrm{nm}$ and the $970-\mathrm{nm}$ optical sources, respectively. Here, $\operatorname{sgn}(x)$ is a signum function and $\mathcal{H}(x)$ is the Hilbert transform. The final chirps are weighted, as shown in Eqs. (4a) and (4b), to ensure that no negative optical intensity is generated. The actual simulation parameters included are $f_{1}=1 \mathrm{MHz}, f_{2}=5 \mathrm{MHz}$, and $T_{c h}=1 \mathrm{~ms}$, while $A_{1}$ and $A_{2}$ are arbitrarily adjusted for the special simulation condition described below.

During the simulation, the sample model shown in Fig. 2 was assumed to contain three distinct PA sources at different locations. ${ }^{21}$ The first PA source was named "noise," and this was the noise that IV-DPAR was not perfectly tuned to suppress. This simulated the potential driver-borne radio frequency (RF) noise in a real imaging setting, which is expected to be compressed at $0 \mu$ s delay time $(\tau)$ during the matched-filter/ pulse compression processing. The second PA source was named "NoiseObj", and this was the noise that IV-DPAR was tuned for differential suppression. This simulated the arterial wall or any unknown local noise at $\tau=5 \mu$ s away from a detector that could interfere with the desired target PA signals. Last, the third PA source was named "TargetObj", and this was the target that IV-DPAR was tuned to detect with the maximum sensitivity and specificity. This simulated a necrotic core in actual atherosclerotic plaque-imaging scenarios. The position of TargetObj was set to be one of the simulation variables to study the possible PA interference effects in FD. As shown in Table 1, the PA signal from TargetObj (cholesterol) was assumed to be weaker than that from NoiseObj (arterial wall) to simulate the special imaging condition where extracting true cholesterol signal was more challenging. While $I_{1}$ and $I_{2}$ were adjusted so that the magnitude ratio of the resulting PA signals was equal to 1 for NoiseObj [by adjusting the abovementioned parameters $A_{1}$ and $A_{2}$ in Eqs. (4a) and (4b)], Gaussian white noise with a factor of 0 or 100 (input SNR of $-40 \mathrm{~dB}$ ) was added to the system to compare the results from an ideal noise-free and more realistic noisy

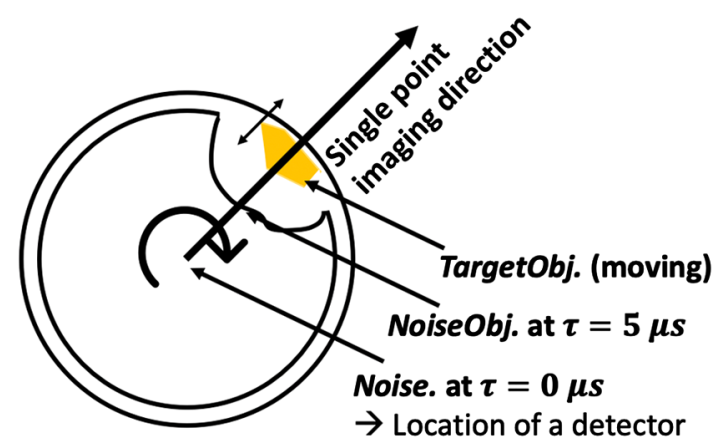

Fig. 2 Atherosclerotic artery model for simulation. ${ }^{21}$ Noise simulated the driver-borne system RF noise that would be isolated near $0-\mu \mathrm{S}$ delay time after the matched-filter/pulse compression processing. NoiseObj simulated the arterial wall that IV-DPAR was tuned to suppress. TargetObj simulated the cholesterols that IV-DPAR was tuned to detect. Assuming that the speed of sound in blood is $\sim 1.5 \mu \mathrm{s} / \mathrm{mm},{ }^{15}$ the delay time information can be directly translated into distance. 
Table 1 Simulation condition: ${ }^{21}$ relative PA signal magnitudes after differential tuning. The magnitude ratio of NoiseObj PA signals was tuned to be 1 for differential suppression.

\begin{tabular}{lccc}
\hline PA Magnitude & $\begin{array}{c}\text { Noise } \\
(0 \mu \mathrm{s})\end{array}$ & $\begin{array}{c}\text { NoiseObj } \\
(5 \mu \mathrm{s})\end{array}$ & $\begin{array}{c}\text { TargetObj } \\
\text { (moving) }\end{array}$ \\
\hline Laser A $(1210 \mathrm{~nm})$ & 0.6 & 1.0 & 0.7 \\
Laser B $(980 \mathrm{~nm})$ & 0.4 & 1.0 & \\
\hline
\end{tabular}

environments. A total of 50 simulation signals were obtained for each measurement condition to evaluate the average. All simulation analysis was performed on a MATLAB 2014 package.

\subsection{IV-DPAR Catheter Prototype}

Two CW diode laser sources of different wavelengths, $\lambda_{1}=$ $1210 \mathrm{~nm}$ (LDX-3210-1210; RPMC, Missouri) and $\lambda_{2}=$ $980 \mathrm{~nm}$ (LDX-3515-980; RPMC, Missouri), were utilized to simultaneously excite target cholesteryl-oleate samples in the differential mode. Both diodes were integrated with our customized fast-modulation drivers (Fast Analog Carlsbad, California) that can modulate the lasers with reliable square waveforms up to $\sim 24 \mathrm{MHz}$. A dual-channel arbitrary waveform generator (33522B; Agilent, California) was employed to modulate the drivers simultaneously with 1-ms-long square wave chirps (frequency sweeps) in the 300 to $14 \mathrm{MHz}$ range at 180-deg phase difference with less than \pm 0.01 - deg deviation. Each channel was coded in a $[1,0,1]$ and $[0,1,1]$ manner, where 0 and 1 represent laser off and on, respectively, so that all possible single-ended (1210 and $980 \mathrm{~nm}$ ) and differential PAR signal combinations could be obtained simultaneously from each location in the sample. The waveform generator was synchronized with a trigger from a National Instruments (NI) computer. While the temperature of each diode was stabilized at $24.5^{\circ} \mathrm{C}$ (room temperature) during the experiments using a TEC controller (Arroyo
5305; Arroyo Instruments, California) with less than $\pm 0.01^{\circ} \mathrm{C}$ deviation, the two fibers carrying each wavelength were coupled into a single fiber with a customized wavelength coupler (WDM-12P-111-980/1210-400/440-QMQMQM-35-555-3A-1, OZ Optics, Ottawa, Ontario, Canada). The single output fiber was then connected to a $400-\mu \mathrm{m}$ fiber with $\sim 34-$ deg cut at the tip (OZ Optics, Ottawa, Ontario, Canada) to deliver the light to the desired location within the vessel by total internal reflection. The beam size of each system was measured to be $\sim 1 \mathrm{~mm}$. The optical power of the $1210-\mathrm{nm}$ laser was set to its maximum ( $\sim 400 \mathrm{~mW})$, and that of the 980-nm laser was arbitrarily tuned to a similar level as part of the differential calibration process. For PA signal acquisition, a customized ultrasonic transducer with $1.5-\mathrm{mm} \times 1.5-\mathrm{mm}$ dimension and $14-\mathrm{MHz}$ center frequency with 10.5- to $16-\mathrm{MHz}$ bandwidth at $-6 \mathrm{~dB}$ (Sunnybrook Research Institute, Toronto, Ontario, Canada) was held by a $2 \mathrm{~mm}^{2} \times 2 \mathrm{~mm}^{2}$ metal holder and was used as a detector.

The transducer and the optical fiber were aligned together and assembled into the catheter prototype. For acoustic coupling, but also for actual endoscopic imaging, the catheter would need to be submerged in blood within the vessel. Since blood has a relatively high refractive index of $\geq 1.41$ at the wavelengths of our interest, ${ }^{23}$ the optical fiber end was covered with a glass cap to provide an air medium that has a lower refractive index than the fiber core $(\sim 1.44)$, ensuring efficient total internal reflection at the fiber tip with no energy loss [Figs. 3(a) and 3(b)]. The diameter of the catheter prototype was $\sim 4 \mathrm{~mm}$. The reasons for its large size include a metal holder and the transducer element itself. For future optimization, such hardware can be easily miniaturized. The received signal was amplified by $40 \mathrm{~dB}$ (5676, Olympus Panametrics, California), and 50 signal records were averaged during the process described in Fig. 1. Data acquisition and signal processing were controlled by a NI card with the sampling rate of $100 \mathrm{MHz}$ (NI PXIe-5122, Texas) and CADIPT-developed LabVIEW software. An atherosclerotic artery phantom was prepared using a porcine artery (Sunnybrook Research Institute, Toronto, Ontario, Canada) and
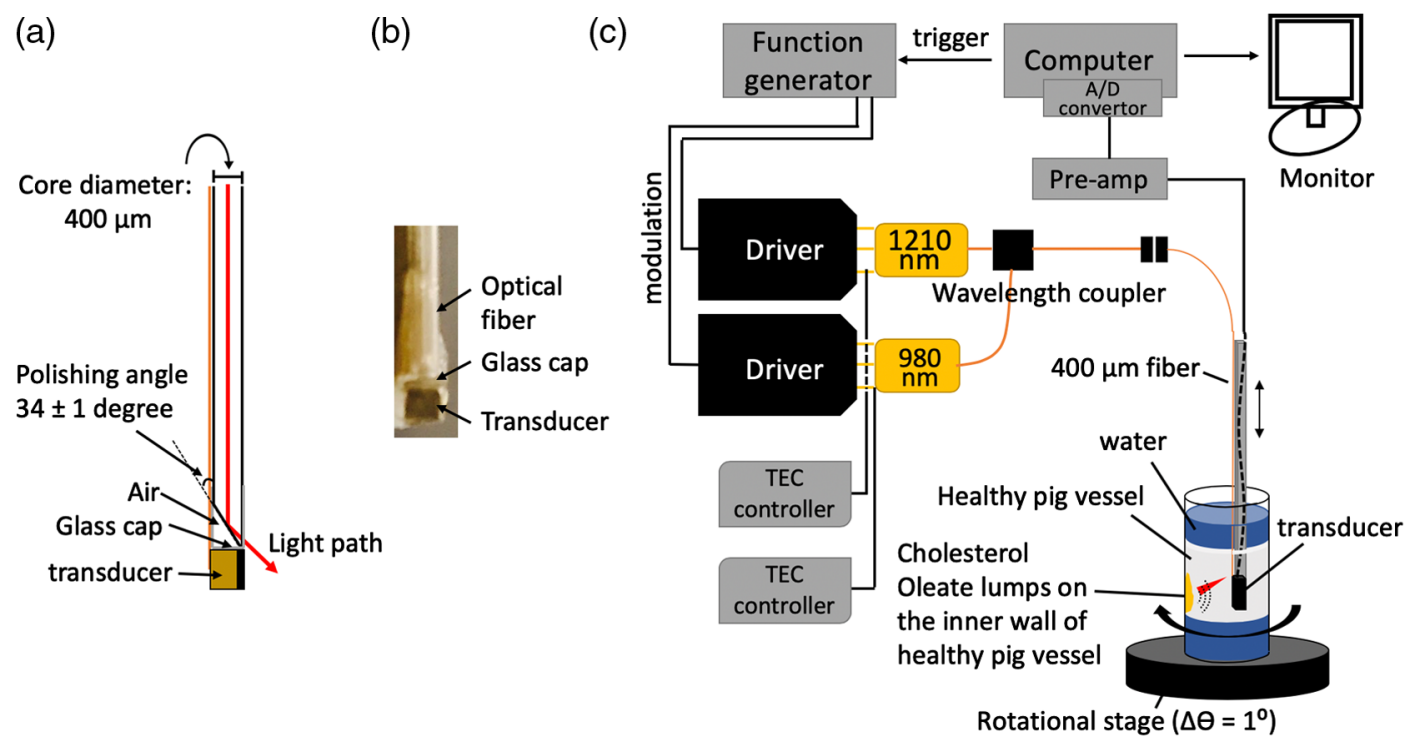

Fig. 3 (a) Design of the IV-DPAR catheter prototype. The detector was placed below the optical fiber so that it was aligned with a light-tissue interaction area in front of it. (b) Picture of the tip of the catheter prototype (front view). (c) Block diagram of the general IV-DPAR experimental setup. The sample stage was designed to allow rotational movement of a loaded sample. 
plaque-mimicking cholesteryl oleate (A11378; Alfa Aesar, Massachusetts). The animal tissue was obtained and handled under the approval of the Sunnybrook Research Ethics Board and the University Health Network Research Ethics Board. To test the differential measurement principles using the built catheter prototype, the $\sim 7 \mathrm{~mm}$-long porcine artery with $\sim 1$-mm diameter was cut open at one end and rotated by 90 deg before rolling back into a cylindrical shape of $\sim 7$-mm diameter. While increasing the inner area of the vessel, such a modification does not affect the biological properties of intima itself. Therefore, this sample was still suitable to test the imaging principles of the system. An arbitrary amount of cholesteryl-oleate powder was melted on a heating plate at $\sim 45^{\circ} \mathrm{C}$, then gently placed on the inner surface of the enlarged artery at two different locations with different thicknesses (thin layer and $\sim 0.7-\mathrm{mm}$ lump). Once the cholesterol samples solidified, the lumen was filled with deionized water for acoustic coupling and the phantom was placed at the center of the rotational stage. While the catheter prototype was fixed near the center, the phantom was rotated 360 deg with $\sim 1$ - deg resolution to obtain a full tomographic cross-sectional image of the inner surface and radial depth profiles. The block diagram of the general experimental setup, including the details of the IV-DPAR measurement window, is depicted in Fig. 3(c).

The same instrumentation and endoscopic transducer were used for IVUS and IV-DPAR image acquisition, greatly facilitating coregistration. During IVUS acquisition, the opticalrelated parts were inactive while the US transducer was used both as an emitter and a receiver. The emitting US signal was modulated with sine-wave chirps in the range from $300 \mathrm{kHz}$ to $24 \mathrm{MHz}$ by the same arbitrary waveform generator. Data acquisition and matched-filter cross-correlation signal processing were performed using the same NI card and the LabVIEW software.

\section{Results and Discussion}

\section{1 $\Delta \varphi$ Scan Simulation: Acoustic Phase Difference Effect on IV-DPAR}

During the optical-to-acoustic energy conversion or general measurements, some degree of phase shift $\left(\varphi_{\text {shift }}\right)$ is unavoidable. $^{21}$ In order to investigate how sensitive the IV-
DPAR signals would be to possible $\varphi_{\text {shift }}$, a full $\Delta \varphi_{\text {acoustic }}$ (or $\Delta \varphi)$ scan, adjusted from 0 deg to 360 deg with 2-deg resolution, was performed on the amplitude channel. For simplicity, the location of TargetObj was fixed at $10 \mu \mathrm{s}$. As shown in Fig. 4(a), when no Gaussian noise was assumed and signal interference between PA sources was minimum due to a large distance between them, NoiseObj suppression was most effective at $\Delta \varphi=180 \mathrm{deg}$, as expected. For noise at $0 \mu \mathrm{s}$, a complete zerobaseline suppression did not materialize because the system was not specifically tuned to suppress this noise source; yet the suppression was most effective at $\Delta \varphi=180 \mathrm{deg}$. When the white Gaussian noise with a factor of 100 , thereby simulating the input SNR of $-40 \mathrm{~dB}$, was added to the simulation to better simulate a reality, the same trend was observed, as shown in in Fig. 4(b). In this case, however, the efficiency of NoiseObj suppression was shown to be quite similar within a small range of 175 deg to 185 deg possibly due to a random $\varphi_{\text {shift }}$ caused by the global system noise. This may mean that IV-DPAR was rather insensitive to inevitable but undesired $\varphi_{\text {shift }}$ during the measurements and effective differential processing can still be achieved in a small range of $\Delta \varphi$ around $180 \mathrm{deg}$.

\subsection{Position Scan Simulation: Signal Interference Effect on IV-DPAR}

In real-imaging scenarios, NoiseObj does not have to be located far from TargetObj since atherosclerotic necrotic cores may be located very close to, or even overlap with, the arterial tissues at the intimal surface depending on the plaque development stage. ${ }^{24}$ Similarly, an undesirable noise (i.e., system RF) may appear at any location with arbitrary magnitude. This type of noise may be very close to or possibly overlap with TargetObj. Therefore, it is important to confirm that the TargetObj PA signal can be fully recovered under such conditions without being altered by local signal interferences. From Fig. 4, it may appear that the TargetObj differential PA signal (red) could also be obtained by simply subtracting the laser B signal from the laser A signal without applying intrinsic wavelength modulation on the two optical sources. This is actually the approach usually pursued when trying to obtain differential PA signals using pulsed optical sources. ${ }^{6,25,26}$ However, the differential PA signal is intrinsically different from signals generated from such a
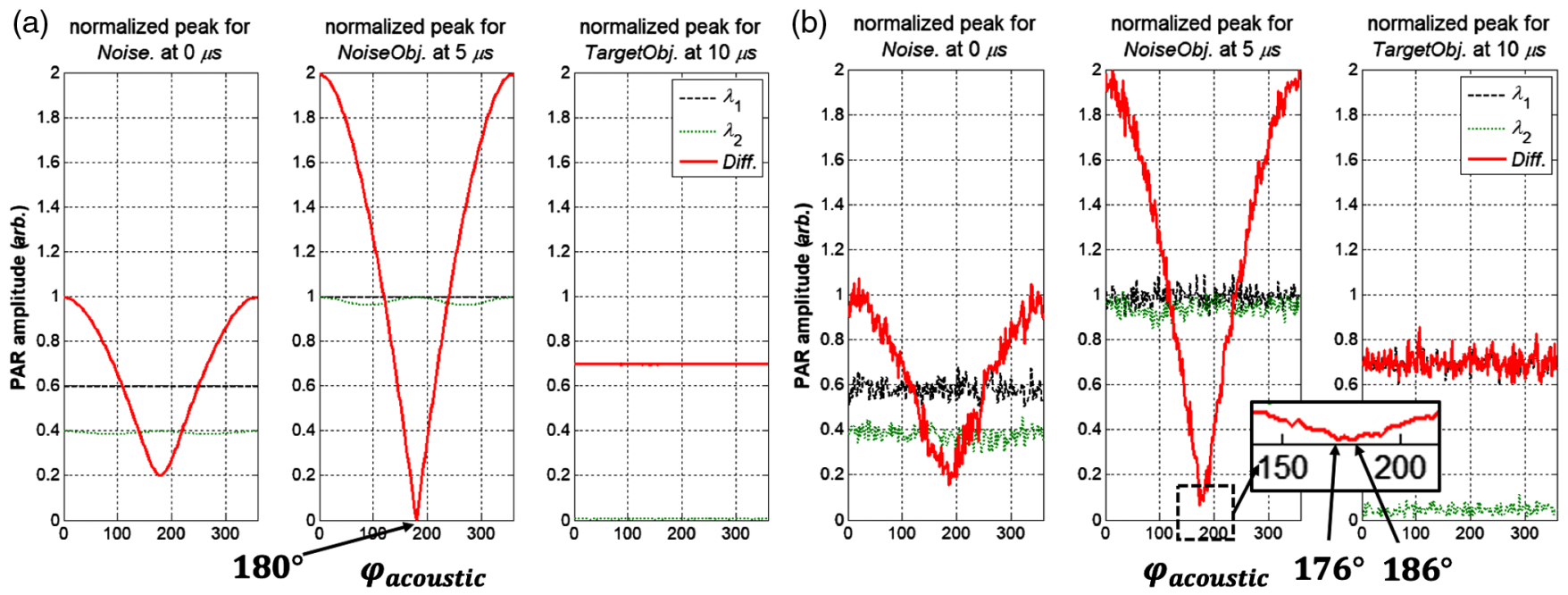

Fig. 4 Full $\Delta \varphi$ scan simulation on the PA amplitude channel. ${ }^{21}$ The three PA sources of the simulation sample were analyzed assuming additive white Gaussian noise with a factor of (a) 0 and (b) 100 . 
simple subtraction (or pseudodifferential) algorithm because it is independent from its individual single-ended components and not affected by local signal interference. To demonstrate this, a position scan simulation was performed on the PA amplitude channel with $\Delta \varphi=180 \mathrm{deg}$ where the position of TargetObj was arbitrarily moved from 2 - to $8-\mu$ s delay time with $0.02-\mu \mathrm{s}$ resolution. The NoiseObj position was fixed at $5 \mu \mathrm{s}$ as before.

Figures 5(a) and 5(b) show how the peak magnitude and the position of the TargetObj PA amplitude signals change, respectively, during the TargetObj position scan in the ideal noise-free environment. ${ }^{21}$ As TargetObj approached and passed through NoiseObj at $5 \mu \mathrm{s}$, the differential PA amplitude was very accurate and reliable in terms of recovering true magnitude and position of the target regardless of the presence of a local interference source: its estimated peak magnitude was always constant, and its estimated peak position always indicated the true position of moving TargetObj with negligible error. However, the single-ended $1210 \mathrm{~nm}$ and the pseudodifferential TargetObj PA channels showed severe error in their estimation near the interfering source. The fact that the same behavior was observed when arbitrary white Gaussian noise with a factor of 100 was considered in the simulation [Figs. 5(c) and 5(d)] proved that such signal fluctuations in both the single-ended $1210 \mathrm{~nm}$ and the pseudodifferential PA channels were caused by unavoidable local signal interferences among multiple PA signals in the region. Such inaccuracy in recovering the true magnitude and position of the TargetObj PA signal can be critical in image analysis as it can lead to generation of artifacts and/ or misinterpretation of important clinical information.

These inaccuracies in the single-ended $1210 \mathrm{~nm}$ and the pseudodifferential PA amplitudes can be further explained by analyzing real and imaginary components of the interfering single-ended 1210-nm PA envelopes in detail. As shown in Fig. 6, when two PA sources are adjacent, the main lobes/ sidelobes of the resulting real and imaginary signals interfere independently from each other. ${ }^{21}$ However, as both real and imaginary components contribute equally to envelope formation, the two envelope signals do not follow a simple linear interference pattern as TargetObj moves through fixed NoiseObj at $5 \mu$ s but repeatedly merge and separate several times before/ after their superposition. This explains why the signal magnitude and position of the single-ended 1210-nm PA mode fluctuated during the interference. This type of error also directly affects the pseudodifferential signal and causes a similar error in that mode. From the simulation parameters used, such errors are found to be dominant within $\mathrm{a} \sim \pm 1 \mu \mathrm{s}$ (or $\sim \pm 1.5 \mathrm{~mm}$ ) range from the interfering source. This value is clearly within the scale of typical human coronary arteries ${ }^{27}$ and, therefore, is considered critical.

(a) normalized TargetObj. peak magnitude scan
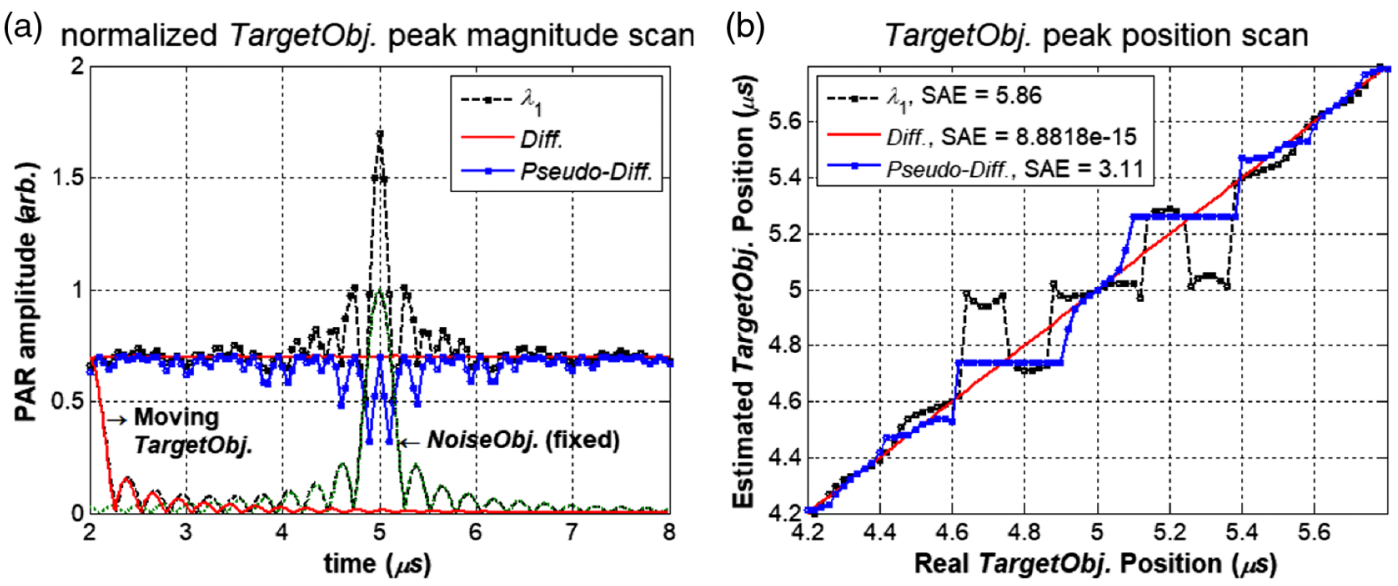

(c) normalized TargetObj. peak magnitude scan

(d) TargetObj. peak position scan

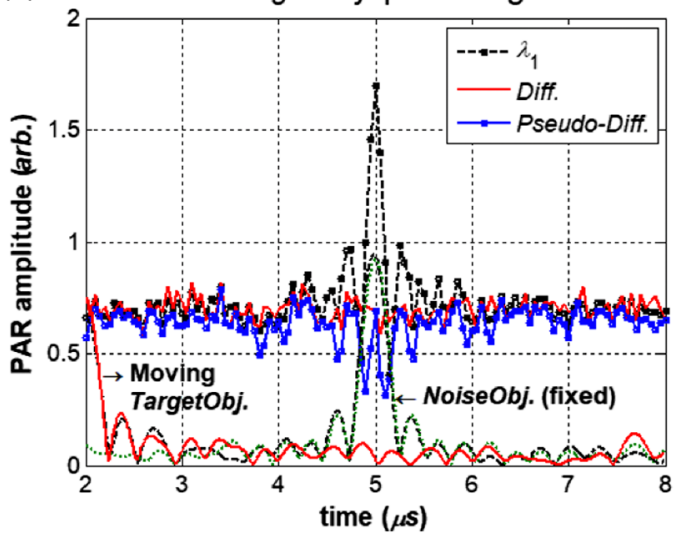

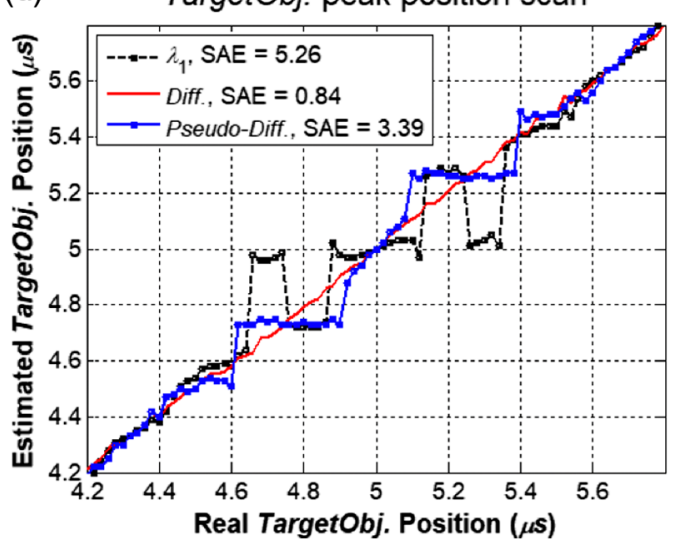

Fig. 5 TargetObj position scan simulation: ${ }^{21}$ Traces of (a) the peak magnitude and (b) the position of TargetObj PA signals from single-ended 1210-nm, differential, and pseudodifferential modes, assuming no white Gaussian noise in the system. The same analysis was done on traces of (c) the peak magnitude and (d) the position of TargetObj signals from the same PA channels assuming white Gaussian noise with a factor of 100 . SAE, sum absolute error. 


\section{normalized single-ended PA interference, NoiseObj. at $5.0 \mu \mathrm{s}$}

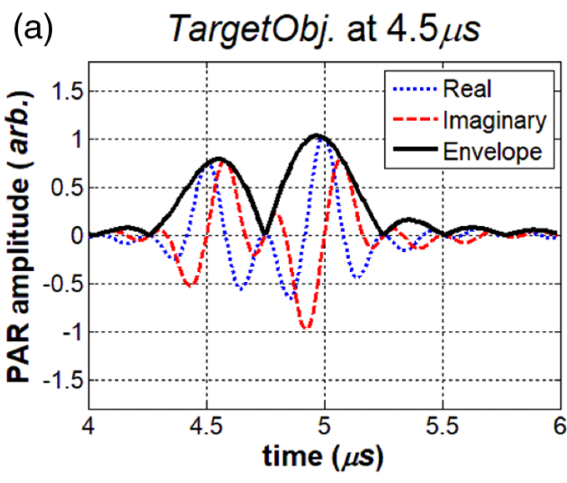

(d)

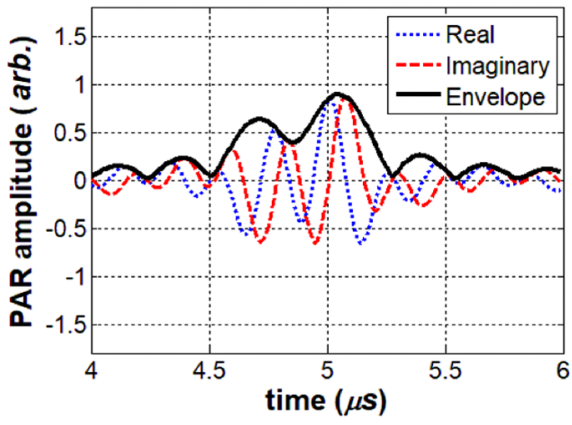

(b)

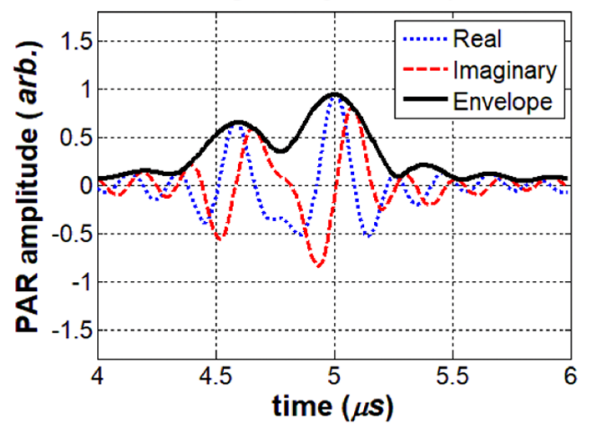

(e)

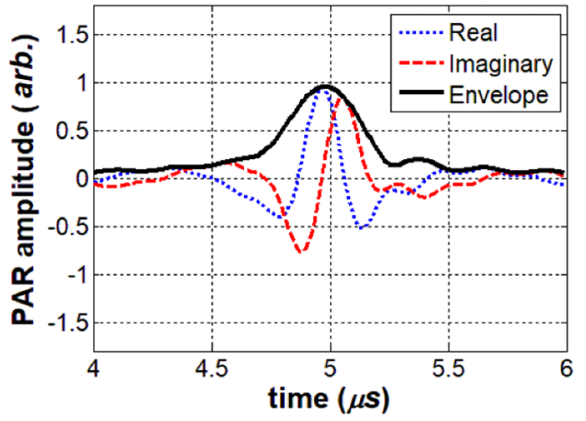

(c)

TargetObj. at $4.7 \mu \mathrm{s}$

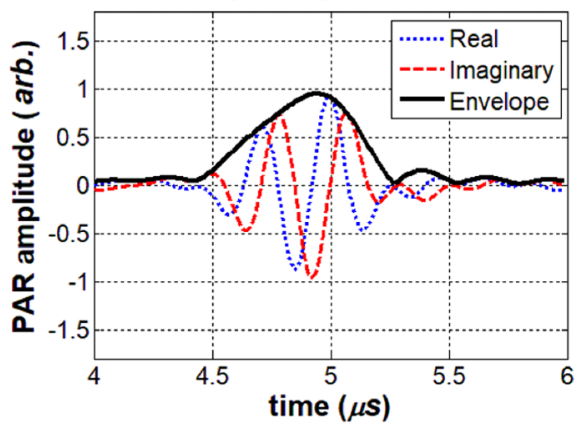

(f)

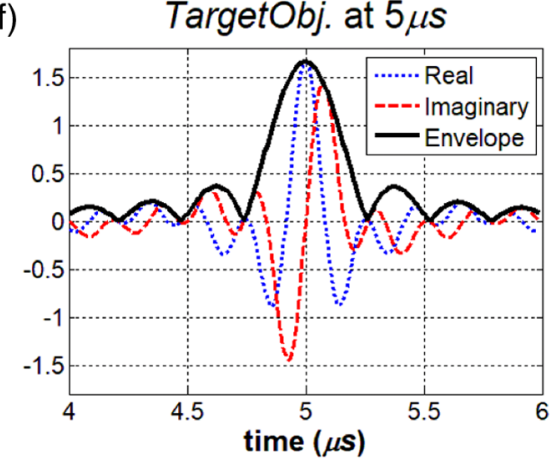

Fig. 6 Sequential events of interference between two single-ended 1210-nm PA envelopes. ${ }^{21}$ As NoiseObj is fixed at $5 \mu \mathrm{s}$, TargetObj approached from distances corresponding to TargetObj at (a) $4.5 \mu \mathrm{s}$, (b) $4.6 \mu \mathrm{s}$, (c) $4.7 \mu \mathrm{s}$, (d) $4.8 \mu \mathrm{s}$, (e) $4.9 \mu \mathrm{s}$, and (f) $5 \mu \mathrm{s}$ (complete overlap).

To resolve this issue, one can always implement a higher chirp frequency range with wider bandwidth (i.e., 1 to $20 \mathrm{MHz}$ ) for optical modulation, which has been the typical approach for detecting smaller details of imaging targets. While SNR and imaging depth were considerably compromised, the improved FWHM axial resolution in this case was shown to reduce the estimation errors of TargetObj PA peak position near NoiseObj in Fig. 7(b). However, as shown in Fig. 7(a), even with such a simulation condition, considerable errors in magnitude estimation were still observed within $\sim \pm 1-\mu$ s range from the interfering source for the abovementioned reasons. ${ }^{21}$ On the other hand, the differential PA signal was clearly unaffected by interferences and could estimate the magnitude and the position of the target very accurately under any condition. This was because IV-DPAR dealt with only one set of real and imaginary components of TargetObj as the NoiseObj components were suppressed from the system before the detection.

While such undesirable signal interferences are very likely to occur during submillimeter scale intravascular plaque imaging, to the best of our knowledge, they have never been thoroughly investigated with conventional time-domain (TD) pulse-based IVPA systems. In many pulse-based studies, only the single wavelength that coincides with the absorption peaks of lipids $(1210$ or $1700 \mathrm{~nm})$ has been utilized to characterize the cholesterol contents of plaques. ${ }^{5,28,29}$ As the bipolar shape of pulsed IVPA signals reduces the probability of differentiating between two depth-wise adjacent chromophores, ${ }^{15}$ the detection accuracy and reliability of such single-wavelength modalities are severely compromised when lipids are located near nontarget chromophores. Various forms of spectroscopic analysis have been tried with pulse-based IVPA systems to differentiate plaques from surrounding tissues, ${ }^{7,30}$ but they require time-consuming multiwavelength measurements for every data acquisition point and each wavelength is still evaluated independently, ignoring the possible effects of signal interferences. While shown to be ineffective in modalities involving FD signal processing, many pulse-based IVPA systems employ the multispectral approach and evaluate the arithmetic difference between the resulting individual PA signals to extract cholesterol information. ${ }^{6,25,26}$ As most pulse-based IVPA systems do not involve FD signal processing, which generates real and imaginary signal components, the interference between two adjacent PA signals would simply be linear. Therefore, this pseudodifferential modality is expected to generate similar interference-free lipid signals as shown in IV-DPAR. However, such an approach in pulsed IVPA is not as reliable as the wavelength-modulated differential method of IV-DPAR for many reasons. Pulse-based IVPA systems suffer from decreased SNR due to the wideband detection, ${ }^{15}$ so multichannel processing would easily amplify the wideband noise along with other types of intrinsic system-induced noise (i.e., electric noise of the transducer). Furthermore, when successive trains of ns-long pulses are applied with large temporal gaps between individual pulses, there can be no synchronous simultaneous signal processing to simply subtract noise in multispectral PA imaging. Under time-sequence conditions, any undesirable time-dependent effects interfere with actual absorber-related signals, especially in in-vivo imaging where the imaging environment changes continuously such as with breathing motion, and thus reliable realtime imaging becomes challenging. In summary, an optimal method to obtain true cholesterol information during endoscopic imaging is to dispose of possible interfering signals before 
(a) normalized TargetObj. peak magnitude scan

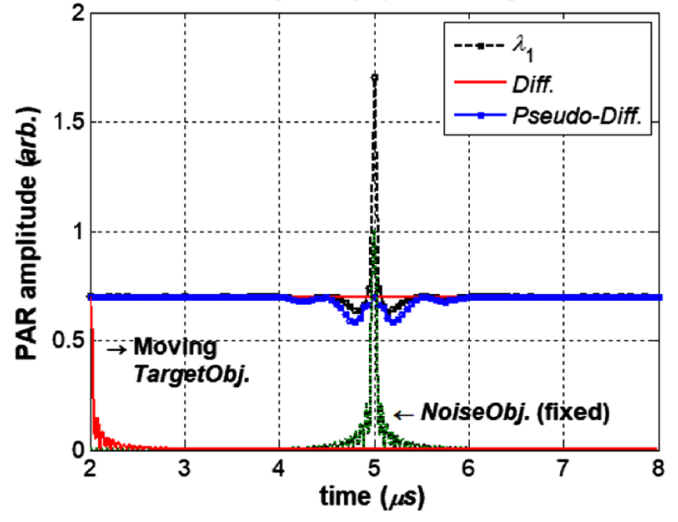

(b)

TargetObj. peak position scan

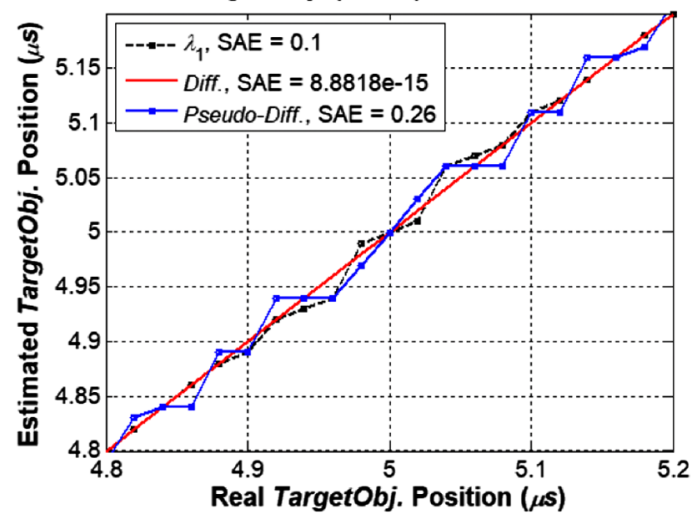

Fig. 7 High-frequency TargetObj position scan simulation: ${ }^{21}$ Traces of (a) the peak magnitude and (b) the position of TargetObj PA signals from single-ended 1210-nm, differential, and pseudodifferential modes, assuming no white Gaussian noise in the system. SAE, sum absolute error.

actual interferences are sensed by the detector. This can be easily achieved using CW-based IV-DPAR through simultaneous wavelength-modulated differential suppression.

\subsection{Baseline Suppression Capability of IV-DPAR}

Under the aforementioned conditions of the phantom experiment, a primary source of interference is found to be currentderived RF signals from the fast-modulating drivers. They are negligible when the diode modulation frequency is low. However, when it increases beyond $\sim 8-\mathrm{MHz}, \mathrm{RF}$ signals that follow the same waveform shape and frequency range as the corresponding driver modulation, start to raise the baseline from the system coherently, thereby concealing the weak PA signals from lipids. These strong wire-/air-transmitted RF signals are picked up by the transducer and are supposed to be compressed into a spike at $0-\mu$ s delay time through the matched filter cross correlation and the pulse compression algorithms in Fig. 1. However, since RF modulation and the diode modulation reference signal do not coincide perfectly in reality, the RF energy leaks to a much longer delay time and becomes problematic for endoscopy imaging where the distance between the target and the receiver is very small.

Figure 8 shows an example of this situation in complementary amplitude and phase channels. When the laser probe encountered the nonlipid area of the sample, the strong RF energy in both channels leaked up to about $2-\mu$ s delay in the single-ended 1210-nm mode. Assuming the speed of sound in water to be about $1.5 \mathrm{~mm} / \mu \mathrm{s},{ }^{15} \mathrm{RF}$ traces that appear up to $2 \mu \mathrm{s}(3.0 \mathrm{~mm})$ would surely interfere with the weak PA signals from plaques as the diameters of the portions of adult human coronary arteries suitable for intravascular imaging range from 2 to $6 \mathrm{~mm} .{ }^{27}$ Therefore, to suppress those RF interferences, the differential method was used. The amplitude ratio and the phase difference of the two lasers were tuned optimally; yet, even when the 1210-nm optical power was set to minimum $(\sim 100 \mathrm{~mW})$, while setting the 980-nm optical power to maximum $(\sim 400 \mathrm{~mW})$, in an attempt to match their background $\mathrm{RF}$ amplitude ratio at $0 \mu \mathrm{s}$, the 1210 -nm driver still generated a stronger RF amplitude than the 980-nm driver. However, as shown in Fig. 8, the 1210- and 980-nm RF signals coincided shortly after $0-\mu$ s delay time and both the differential amplitude and the differential phase signals were successfully suppressed to below the normal system baseline when they reach $\sim 1 \mu \mathrm{s}$.

\subsection{IV-DPAR Signal of the Cholesterol Sample 1: Thin Layer}

For the first sample, cholesterol was gently rubbed around the inner surface of the porcine intima while it was still in the
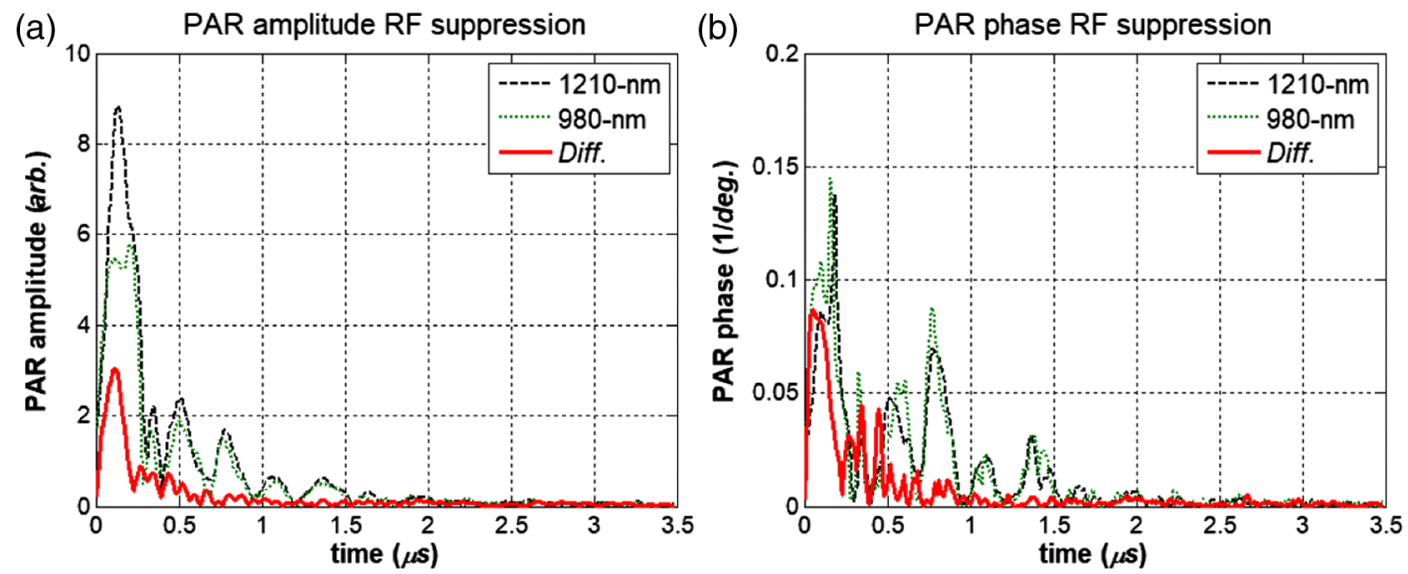

Fig. 8 PAR cross-correlation baseline suppression capability of IV-DPAR in (a) amplitude and (b) phase channel. 
(a) PAR amplitude on C1 (1210-nm highlighted)

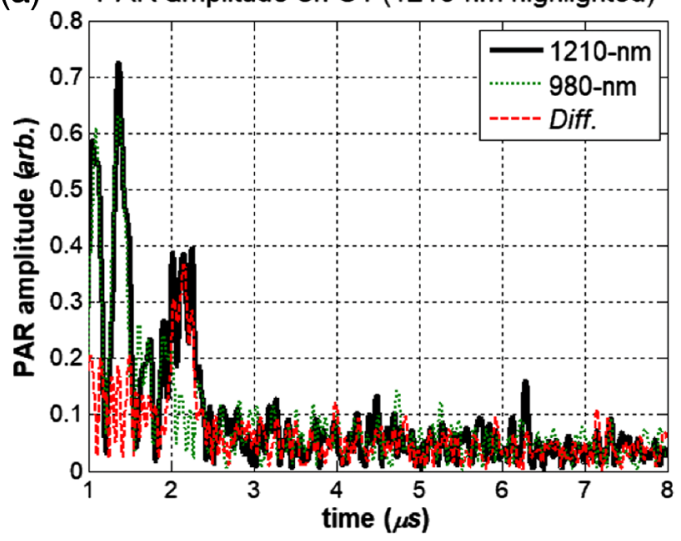

(c) PAR phase on C1 (1210-nm highlighted)

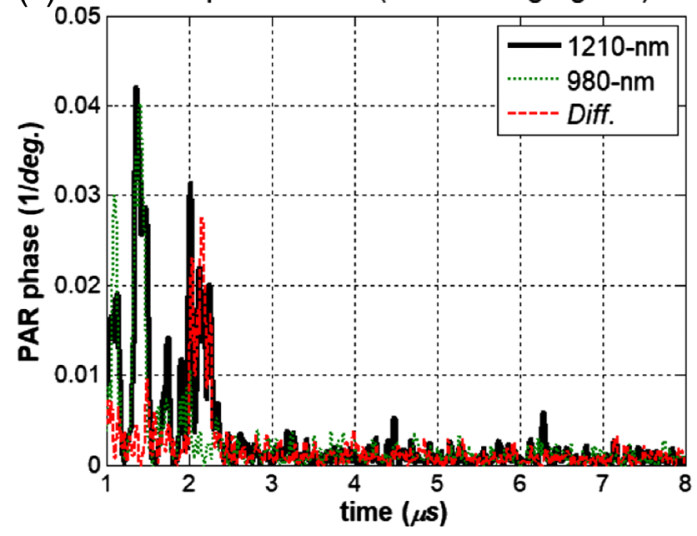

(b) PAR amplitude on $\mathrm{C} 1$ (Diff. highlighted)

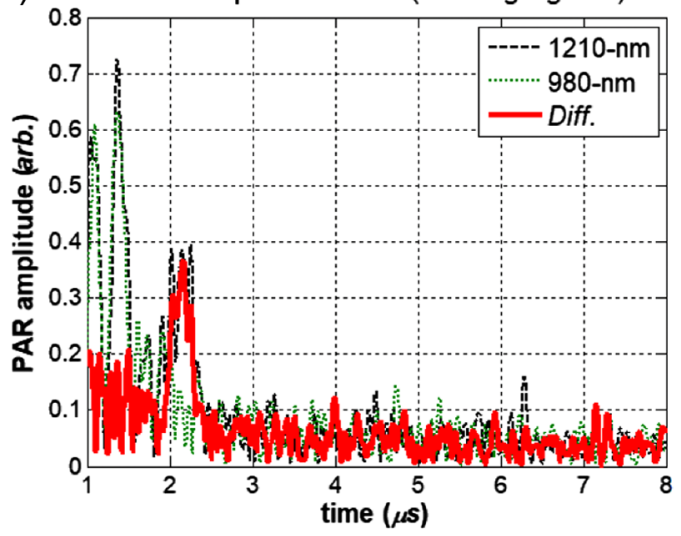

(d)

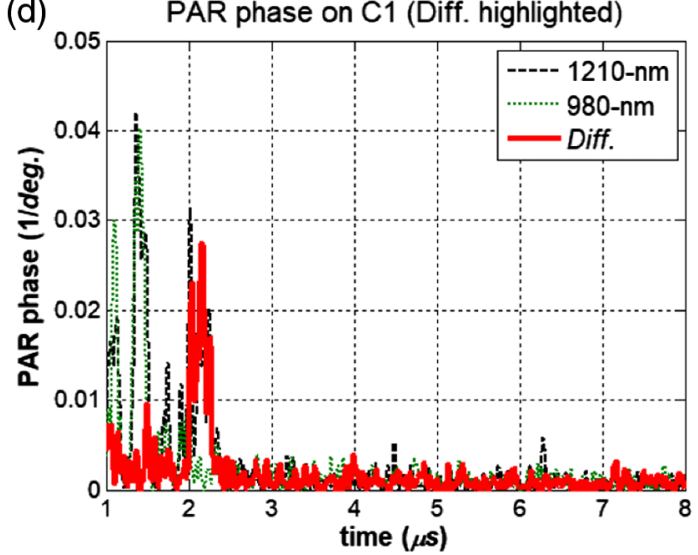

Fig. 9 PAR signals from C1, a thin layer of cholesterol. (a) 1210-nm amplitude, (b) differential amplitude, (c) 1210-nm phase, and (d) differential phase signal. C1, Cholesterol sample 1; C2, Cholesterol sample 2.

gel-like state. After it solidified, it became a thin layer of lipid on the tissue, which was barely visible to the naked eye. Figure 9 depicts the PAR amplitude and phase signal traces of the cholesterol sample 1 obtained by the single-ended PAR and the IV-DPAR modes for comparison [1210-nm and differential signals are highlighted in (a), (c) and (b), (d), respectively]. Both the PA single-ended $1210 \mathrm{~nm}$ and differential systems in two different channels (amplitude and phase) detected the arbitrary cholesterol sample 1 at nearly $2.1 \mu \mathrm{s}$, but the contrast difference is clearly due to the baseline suppression capability of IVDPAR. As shown elsewhere, ${ }^{15}$ the PAR phase channel generally exhibits greater SNR when compared to the corresponding amplitude channel due to its lower system baseline noise. In addition, as shown in Figs. 9(c) and 9(d), phase signals from cholesterol are more temporally confined and therefore show better axial resolution than corresponding amplitude signals, as the phase peak occurs between the real and the imaginary components of the cross-correlated PA signal during the image processing in Fig. 1. ${ }^{15}$ Comparing Figs. 9(b) and 9(d) and assuming the speed of sound in the medium to be about $1.5 \mathrm{~mm} / \mu \mathrm{s}$, axial resolutions of amplitude and phase channels at this position of the sample were $\sim 0.44$ and $\sim 0.30 \mathrm{~mm}$, respectively.

\subsection{IV-DPAR Signal of the Cholesterol Sample 2: Thick Lump}

To test the IV-DPAR ability to estimate lipid thickness and image radial depth profiles, a second cholesteryl-oleate sample, a single drop of the heated gel-like state cholesterol, was placed on the inner surface of the porcine intima. As it solidified over time, it appeared as a visible lump of cholesterol adhered to the tissue. After the imaging experiment, this lump was carefully detached from the tissue and its thickness was measured by a mechanical caliper to be about $0.7 \mathrm{~mm}$.

Figure 10 depicts the PAR amplitude and phase signals of the cholesterol sample 2 that were generated by the single-ended PA and the IV-DPAR modes [1210-nm and differential signals are highlighted in (a),(c) and (b),(d), respectively]. From the singleended 1210-nm PAR amplitude in both channels, it was very difficult to even identify the signals generated from cholesterol because of RF interference. However, the two cholesterol-specific peaks corresponding to the front and back boundaries of the lump emerged clearly in the differential modes with excellent SNR due to the baseline suppression capability of IV-DPAR.

The single-ended 1210-nm signals showed some signal suppression at $\sim 2.5 \mu \mathrm{s}$ in both channels. This was possibly due to the residual local RF energy that may interfere with the cholesterol peaks destructively. On the other hand, the differential channel was immune to such an effect since the background was already suppressed intrinsically in the acoustic domain. The thickness of the cholesterol lump could be easily estimated from the differential PA signals. The delay time difference between the two peaks was measured to be $\sim 0.49 \mu$ s in both amplitude and phase channels. Using the aforementioned speed of sound in water, the delay time difference could be directly converted to $\sim 0.73 \mathrm{~mm}$ in thickness, which was in excellent agreement with 

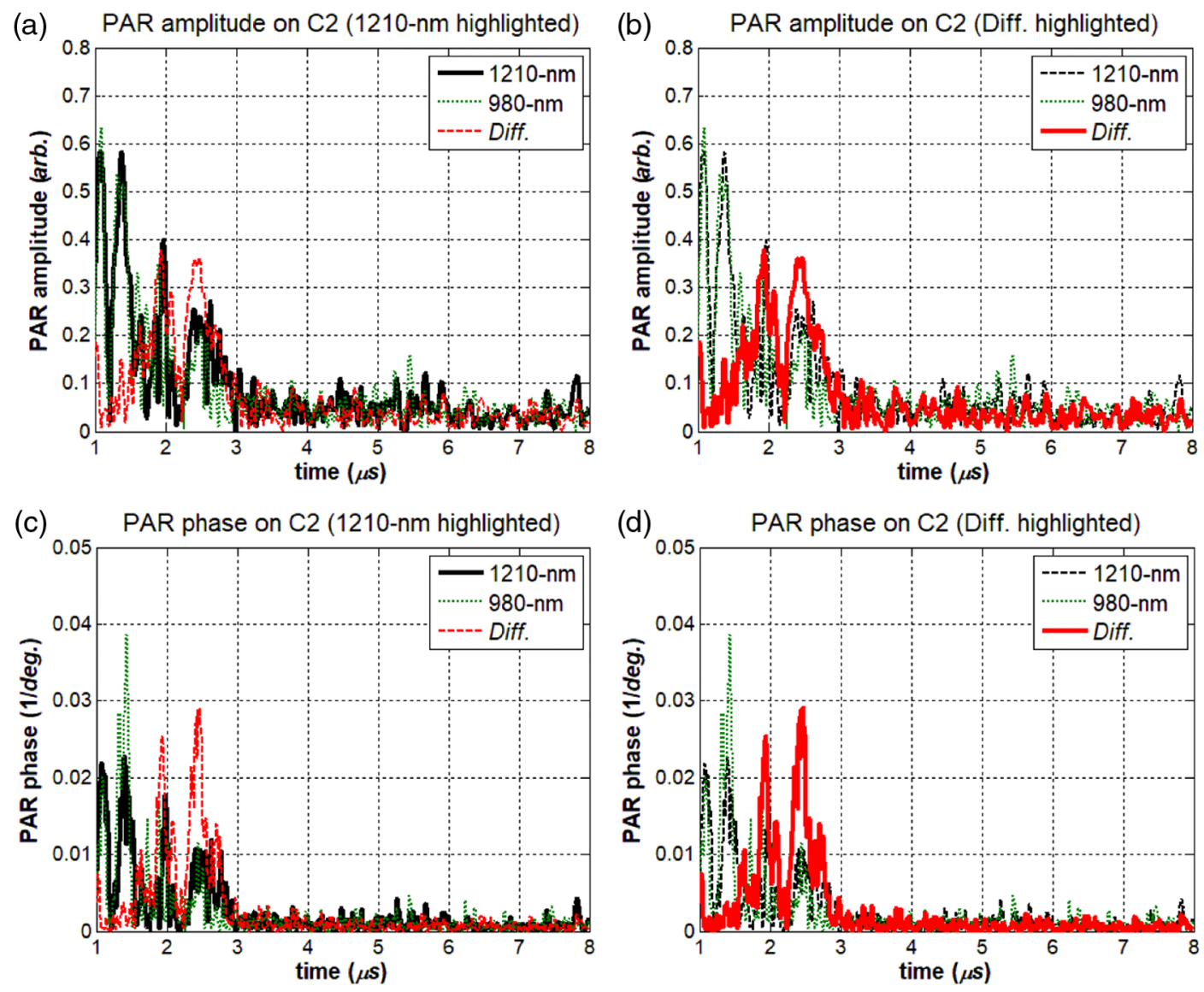

Fig. 10 PAR signals from C2, a visible lump with 0.7-mm thickness. (a) 1210-nm amplitude, (b) differential amplitude, (c) 1210-nm phase, and (d) differential phase. C1, Cholesterol sample 1 and C2, Cholesterol sample 2.

the actual thickness of $\sim 0.7 \mathrm{~mm}$ as was independently measured using the mechanical caliper.

\subsection{Coregistration of IV-DPAR and IVUS}

When such signal traces are collected from full 360-deg angles, a complete circular cross-sectional image of the phantom can be obtained. Since the IV-DPAR system is highly specific for cholesterol molecules, a widely available IVUS can be coregistered easily to provide extra detailed structural information of the background arteries. As IV-DPAR and IVUS share the same transducer and instrumentation for their signal acquisition process, such coregistration is very efficient, as well as cost- and time-effective.

Figure 11(a) depicts the IVUS image of the same plaque phantom with the 14-MHz detector. As IVUS is typically performed at around $40-\mathrm{MHz}$ modulation range, our IVUS image exhibited low resolution. While it can be improved by optimizing the frequency range, it was still acceptable for the coregistration purposes as it could provide the structural background information and the entire depth profile of the porcine arteries. As expected, IVUS was unable to detect the two cholesterol clusters as the US mode lacks sensitivity and specificity to cholesterol. It shows fewer details of the bottom-left region than the other areas due to transducer alignment problems.

Amplitude and phase sets of PA images were simultaneously obtained in single-ended and differential modes and coregistered with the corresponding IVUS image in Figs. 11(b) and 11(c). For the single-ended PA images, the background was arbitrarily subtracted for more meaningful comparison as they were dominated by the residual RF interference.

When coregistered with the IVUS image, the differential PAR mode detected the two cholesterol samples with excellent accuracy and exhibited superior contrast and higher axial resolution than the single-ended 1210-nm mode. As discussed in the earlier sections, the single-ended 1210-nm PA mode showed some false cholesterol cluster regions and signal suppression due to residual local signal interference. As the differential system was specifically tuned to suppress the prevailing RF interference, however, the IV-DPAR modality provided reliable quantitative information on cholesterol in two complementary channels with improved SNR and dynamic range. With no lipid absorption, the single-ended 980-nm PA image did not show any contrast from the cholesterol clusters as expected; yet it showed many residual RF traces. While the differential PA phase channel provided more localized images of cholesterol clusters than the amplitude channel due to more confined signal traces, complementary information from both channels further enhanced the reliability of atherosclerotic plaque detection.

A direct comparison between the single-ended $1210 \mathrm{~nm}$ and the differential modes was possible in Figs. 11(b) and 11(c) because the single-ended images were constructed from the sets of postprocessed data. While such postprocessing improves the single-ended PA image quality, in general it can be risky when the system RF and the desired lipid signals overlap, even partially. As the RF signal is simply subtracted during 


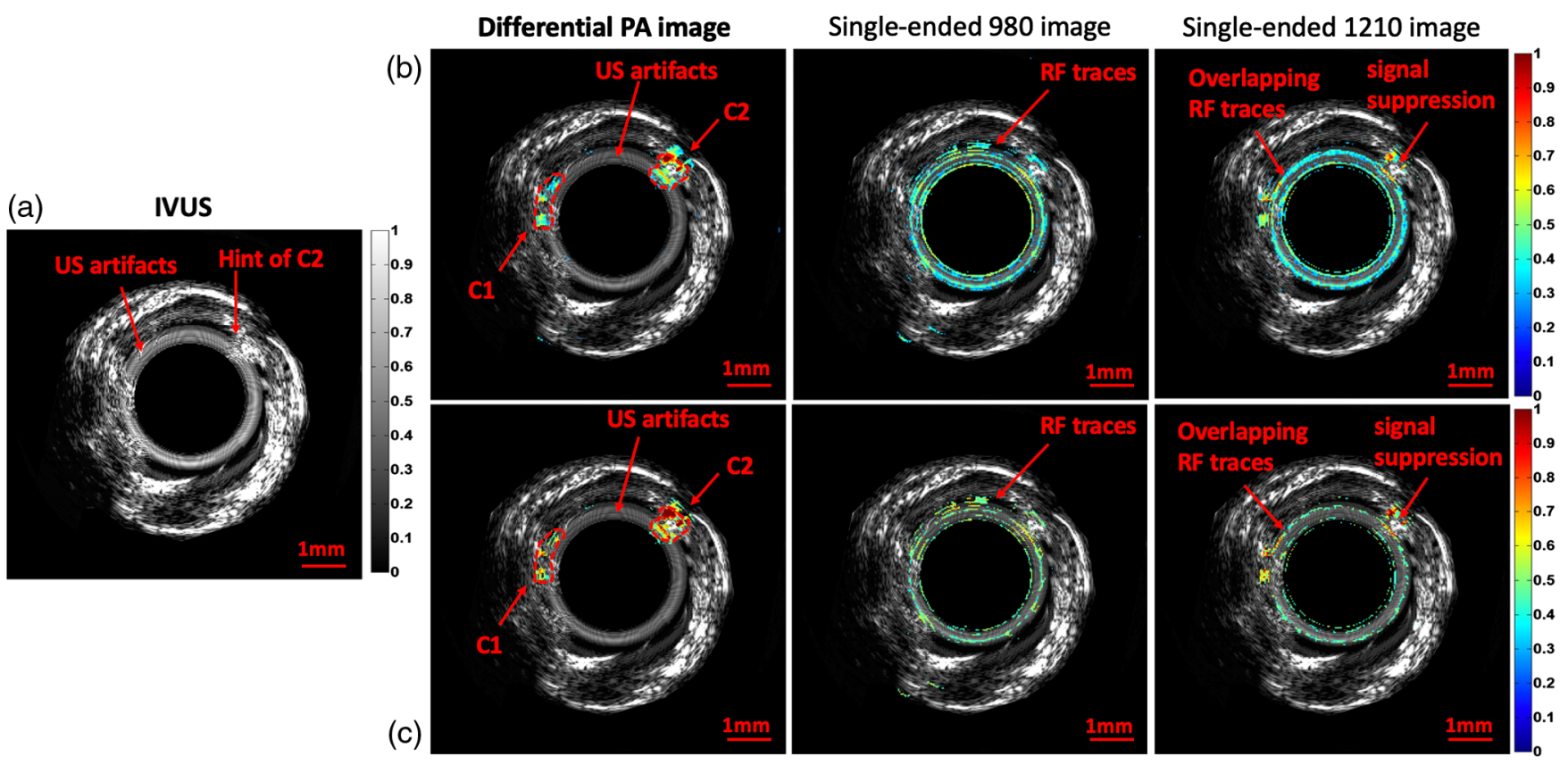

Fig. 11 (a) Normalized 14-MHz IVUS image of the atherosclerotic artery phantom by IVUS at 14-MHz. (b) Normalized PAR amplitude and (c) phase images of IV-DPAR, single-ended 980-nm PAR and singleended 1210-nm PAR modes. The same endoscopic transducer and instrumentation were shared by IVUS and IV-DPAR for coregistration. C1, Cholesterol sample 1 and C2, Cholesterol sample 2.

postprocessing, the overlapped cholesterol information may also become lost. Furthermore, such postprocessing slows down the image processing algorithms in general and, therefore, may limit the real-time imaging capability of the technology. On the other hand, IV-DPAR does not require extra postprocessing because it intrinsically suppresses the RF interference.

Using compact, low-cost, and low-power CW lasers as optical sources, IV-DPAR can be suitable for clinical uses. Furthermore, due to high pulse-repetition frequency (PRF) of CW lasers $(\sim 10 \mathrm{kHz})$, IV-DPAR is much more likely to make dynamic PA imaging practical with reduced motion artifacts compared to its pulse-based counterparts with PRF $<50 \mathrm{~Hz}$. The maximum frame rate that IV-DPAR can achieve would mainly depend on the number of signal averages during the processing in Fig. 1. Focusing on the baseline suppression capability of the differential system, this study is not mainly concerned with system frame rate optimization for real-time imaging purposes. This aspect of IV-DPAR development, along with effective catheter miniaturization, will need to be further investigated in follow-up studies.

In this preliminary study where a simplified model of atherosclerosis was used, the primary source of PA signal was cholesteryl oleates only. However, in case of real in-vivo atherosclerosis imaging, there can be many other potential PA sources for the chosen wavelengths such as blood and calcium that can all contribute to the final PA signals of the system. The conventional single-ended PA imaging modality then may become problematic since its signal would be affected by the total absorption of light from nearby chromophores. However, the IV-DPAR mode with its multispectral imaging capability would be able to simultaneously generate the two single-ended PA signals at different wavelengths and the differential PA signal that is mostly sensitive to light absorption by cholesterol and less so to other overlapping but lower absorption wavelengths, thereby enhancing the IV-DPAR endoscopic imaging technique.

\section{Conclusions}

In this study, the theoretical background and experimental validation of IV-DPAR are presented as a reliable CW-based PA imaging method that can detect true cholesterol signals in plaques. Unlike the conventional PAR and other single-ended pulse-based IVPA techniques, this modality is only sensitive and specific to spectroscopically defined targets without being affected by undesirable local interference with noise signals. This modality is unlike conventional pulse-based multispectral PA modalities that rely on simple subtraction algorithms of sequentially processed signals. When coregistered with IVUS, IV-DPAR could reveal a complete cross-sectional image of the phantom that delivers accurate location and quantitative radial depth-profile of two arbitrary plaque models.

\section{Disclosures}

Authors declare no conflicts of interest.

\section{Acknowledgments}

SS. S. C. gratefully acknowledges the support of the Natural Sciences and Engineering Research Council of Canada (NSERC) for an Alexander Graham Bell Canada Graduate Scholarship-Doctoral (CGS-D) and the government of Ontario for a Queen Elizabeth II Graduate Scholarship in Science and Technology (QEII-GSST). A. M. acknowledges an NSERC Discovery Award and the Canada Research Chairs Program. A. M. and S. F. F. are also grateful to the Canadian Institutes of Health Research (CIHR) and NSERC for a Collaborative Health Research Project (CHRP) grant. 


\section{References}

1. R. Virmani et al., "Lessons from sudden coronary death: a comprehensive morphological classification scheme for atherosclerotic lesions," Arterioscl. Throm. Vas. 20(5), 1262-1275 (2000).

2. C. P. Sparrow and J. Olszewski, "Cellular oxidation of low density lipoprotein is caused by thiol production in media containing transition metal ions," J. Lipid Res. 34(7), 1219-1228 (1993).

3. J. M. Tarkin et al., "Imaging atherosclerosis," Circ. Res. 118(4), 750-769 (2016).

4. P. Libby, P. M. Ridker, and A. Maseri, "Inflammation and atherosclerosis," Circulation 105(9), 1135-1143 (2002).

5. S. Hirano et al., "Aortic atherosclerotic plaque detection using a multiwavelength handheld photoacoustic imaging system," Proc. SPIE $\mathbf{9 7 0 8 ,}$ 98084Y (2016).

6. T. J. Allen et al., "Spectroscopic photoacoustic imaging of lipid-rich plaques in the human aorta in the 840 to $1400 \mathrm{~nm}$ wavelength range," J. Biomed. Opt. 17(6), 061209 (2012).

7. K. Jansen et al., "Photoacoustic imaging of human coronary atherosclerosis in two spectral bands," Photoacoustics 2(1), 12-20 (2014).

8. S. Sethuraman et al., "Spectroscopic intravascular photoacoustic imaging to differentiate atherosclerotic plaques," Opt. Express 16(5), 3362-3367 (2008).

9. T. Roleder and W. Wojakowski, "Intravascular ultrasound, optical coherence tomography and near infrared spectroscopy," Cor Et Vasa 57(6), e439-e445 (2015).

10. C. M. Gardner et al., "Detection of lipid core coronary plaques in autopsy specimens with a novel catheter-based near-infrared spectroscopy system," JACC-Cardiovasc. Imaging 1(5), 638-648 (2008).

11. S. E. Nissen and P. Yock, "Intravascular ultrasound: novel pathophysiological insights and current clinical applications," Circulation 103(1), 604-616 (2001).

12. H. M. Garcia-Garcia, M. A. Costa, and P. W. Serruys, "Imaging of coronary atherosclerosis: intravascular ultrasound," Eur. Heart J. 31(20), 2456-2469 (2010).

13. G. J. Tearney, I. K. Jang, and B. E. Bouma, "Optical coherence tomography for imaging the vulnerable plaque," J. Biomed. Opt. 11(2), 021002 (2006).

14. S. Telenkov et al., "Frequency domain photoacoustic correlation (radar) imaging: a novel methodology for non-invasive imaging of biological tissues," Proc. SPIE 8223, 82231J (2012).

15. B. Lashkari and A. Mandelis, "Comparison between pulsed laser and frequency-domain photoacoustic modalities: signal-to-noise ratio, contrast, resolution, and maximum depth detectivity," Rev. Sci. Instrum. 82(9), 094903 (2011).

16. S. Telenkov and A. Mandelis, "Photothermoacoustic imaging of biological tissues: maximum depth characterization comparison of timeand frequency-domain measurements," J. Biomed. Opt. 14(4), 044025 (2009).

17. A. Mandelis, "Imaging cancer with photoacoustic radar," Phys. Today 70(5), 42-48 (2017).

18. S. S. S. Choi et al., "Wavelength-modulated differential photoacoustic spectroscopy (WM-DPAS): theory of a high-sensitivity methodology for the detection of early-stage tumors in tissue," Int. J. Thermophys. 36(5-6), 1305-1311 (2014)

19. S. S. S. Choi et al., "Wavelength-modulated differential photoacoustic spectroscopy (WM-DPAS) for noninvasive early cancer detection and tissue hypoxia monitoring," J. Biophotonics 9(4), 388-395 (2016).
20. S. S. S. Choi et al., "Wavelength-modulated differential photoacoustic radar imager (WM-DPARI): accurate monitoring of absolute hemoglobin oxygen saturation," Biomed. Opt. Express 7(7), 2586-2596 (2016).

21. S. S. S. Choi et al., "Frequency-domain differential photoacoustic radar: theory and simulation for ultra-sensitive cholesterol imaging," Proc. SPIE 10878, 1087812 (2019).

22. S. Telenkov, R. Alwi, and A. Mandelis, "Photoacoustic correlation signal-to-noise ratio enhancement by coherent averaging and optical waveform optimization," Rev. Sci. Instrum. 84(10), 104907 (2013).

23. M. Friebel and M. Meinke, "Model function to calculate the refractive index of native hemoglobin in the wavelength range of 250-1100 nm dependent on concentration," J. Biomed. Opt. 11(3), 034021 (2006).

24. R. J. Jones, Ed., Evolution of the Atherosclerotic Plaques, University of Chicago Press, Chicago, Illinois (1963).

25. S. Shang et al., "Simultaneous imaging of atherosclerotic plaque composition and structure with dual-mode photoacoustic and optical coherence tomography," Opt. Express 25(2), 530-539 (2017).

26. J. Mark et al., "Dual-wavelength 3D photoacoustic imaging of mammalian cells using a photoswitchable phytochrome reporter protein," Commun. Phys. 1, 3 (2018).

27. J. T. Dodge et al., "Lumen diameter of normal human coronary arteries. Influence of age, sex, anatomic variation, and left ventricular hypertrophy or dilation," Circulation 86(1), 232-246 (1992).

28. J. Hiu et al., "Real-time intravascular photoacoustic-ultrasound imaging of lipid-laden plaque at speed of video-rate level," Proc. SPIE 10062, 100640T (2017).

29. D. van der Laan et al., "Real-time intravascular ultrasound and photoacoustic imaging," IEEE Trans. Ultrason. Ferr. 64(1), 141-149 (2017).

30. B. Wang et al., "Intravascular photoacoustic imaging," IEEE J. Sel. Top. Quantum. 16(3), 588-599 (2010).

Sung Soo Sean Choi is a PhD candidate at the University of Toronto, Ontario, Canada. He received his MASc degree in biomedical engineering from the University of Toronto in 2015, and his BSc degree in biomedical sciences from the University of Waterloo, Ontario, Canada, in 2012. His research interest is in developing novel medical imaging technology for early disease detection. $\mathrm{He}$ is a student member of SPIE.

Bahman Lashkari received his $\mathrm{PhD}$ from the Center for Advanced Diffusion-Wave Technologies (CADIFT), University of Toronto, Toronto, Ontario, Canada, working on frequency-domain photoacoustic imaging in 2011. Currently, he is a research associate at the CADIFT, where he works on several photoacoustic and ultrasound projects. His research interests include medical imaging and tissue characterization.

Andreas Mandelis is a full professor of mechanical and industrial engineering, and of electrical and computer engineering, at the Institute of Biomaterials and Biomedical Engineering, University of Toronto. He is also director of the Center for Advanced DiffusionWave and Photoacoustic Technologies (CADIPT) at the University of Toronto. He has published more than 400 scientific papers in refereed journals and 190 proceedings papers. He has received numerous national and international prizes and awards and has several patents in biothermophotonics and biophotoacoustic tissue imaging.

Biographies of the other authors are not available. 